\title{
Uma proposta para integrar a gestão do conhecimento ao Balanced Scorecard
}

\author{
Simone de Cássia Silva \\ Doutorado em Engenharia de Produção pela Universidade Federal \\ de Santa Catarina - UFSC \\ Professora da Universidade Estadual de Santa Cruz - UESC \\ Departamento de Ciências Exatas e Tecnológicas. Rod. Ilhéus-Itabuna, Km 16. \\ Ilhéus/BA. CEP: 45662-000 \\ E-mail:scassia@gmail.com \\ Paulo Mauricio Selig \\ Doutorado em Engenharia pela Universidade Federal de Santa Catarina - UFSC \\ Professor da Universidade Federal de Santa Catarina - UFSC \\ Departamento de Eng. de Produção e Sistemas. UFSC. Florianópolis/SC. CEP: 88040- \\ E-mail: selig@egc.ufsc.br \\ José Luis Duarte Ribeiro \\ Doutorado em Engenharia pela Universidade Federal do Rio Grande do Sul - UFRGS \\ Professor da Universidade Federal do Rio Grande do Sul - UFRGS \\ Av. Osvaldo Aranha 99, 5ำ Andar, sala LOPP. Porto Alegre/RS. CEP: 90035-190 \\ E-mail: ribeiro@producao.ufrgs.br
}

\section{RESUMO}

Este artigo apresenta um modelo para auxiliar no alinhamento da gestão do conhecimento à gestão estratégica das organizações. $O$ modelo foi desenvolvido através de uma pesquisa exploratória de natureza qualitativa. A principal contribuição e originalidade do modelo é sua estrutura para conversão do conhecimento individual em coletivo, do conhecimento estratégico em operacional e do conhecimento tácito em explícito, de forma bilateral. O modelo enfatiza a identificação, seleção, disseminação e avaliação central de informações estratégicas como atividades sistêmicas. Trata-se de uma proposta relevante àqueles que visam investigar novos métodos para intervir na complementaridade da gestão do conhecimento e da gestão estratégica, com vistas a contribuir para a melhoria de aspectos econômicos, mercadológicos, tecnológicos, operacionais, sociais ou ambientais.

Palavras-chave: Gestão do conhecimento. Gestão estratégica. Balanced Scorecard.

A proposal to integrate the management of knowledge to Balanced Scorecard 


\section{ABSTRACT}

This paper presents the development of a model to support the alignment of knowledge management to the strategic management of organizations. The research carried out here has qualitative and exploratory characteristics. The main contribution and the genuine aspects of the model is its structure, which allows the bilateral conversion of individual into group knowledge, of strategic into operational knowledge, and of tacit into explicit knowledge. The model emphasizes the identification, selection, dissemination and evaluation of central strategic information as systemic activities. It can be considered as a relevant proposal to those investigating new methods considering knowledge management and strategic management as complementary activities and thus interfering in the process. The proposed model contributes for the improvement of economic, market, technological operational, social or environmental aspects within the industrial scenario.

Key words: Knowledge management. Strategic management. Balanced Scorecard.

\section{INTRODUÇÃO}

Com o crescente e rápido avanço das informações que permeiam as diversas redes de relacionamento - tanto públicas como privadas - é relevante o gerenciamento constante do conhecimento. Entende-se que o conhecimento deve desempenhar um papel central para a conquista da vantagem competitiva, em termos econômico e financeiro, de mercado, dos processos e dos aspectos sociais, ambientais e tecnológicos. Nesse contexto, este artigo apresenta uma metodologia de acompanhamento da conversão do conhecimento individual em coletivo, do conhecimento estratégico em operacional e do conhecimento tácito em explícito nas companhias, de forma contínua e bilateral. A metodologia proposta visa o alinhamento das diretrizes da rede estratégica de relacionamento entre os variados stakeholders, visando gerenciar uma empresa sob uma vertente cognitiva e conectiva.

Este artigo parte de um silogismo dialético a ser considerado: o alinhamento da gestão do conhecimento (GC) à estratégia organizacional. A partir desta proposição, é relevante a maximização dos esforços na tentativa de direcionar as ações coletivas dos agentes organizacionais aos objetivos estratégicos das organizações.

Assim, esse artigo caracteriza um esforço de pesquisa orientado a responder a seguinte questão: como alinhar, de forma dinâmica, a gestão do conhecimento à gestão 
estratégica, visando promover a conversão simultânea do conhecimento individual em coletivo, do conhecimento estratégico em operacional e do conhecimento tácito em explícito? O direcionamento da resposta parte da pré-condição da implantação do sistema estratégico Balanced Scorecard (BSC) na organização.

Pautando-se em Gil (1999), o método dialético fundamenta-se na proposta de Hegel, na qual as contradições se transcendem dando origem a novas contradições que passam a requerer solução. É um método de interpretação dinâmica e totalizante da realidade. Considera que os fatos não podem ser considerados fora de um contexto social, político e econômico.

Segundo Dagnino (2002, p. 96), a construção de um modelo é essencial para se compreender o funcionamento do sistema que integra a GC ao BSC, desta forma, é possível atuar sobre cada uma de suas características. Assim, considerando a modelagem sistêmica, as características propostas para a melhoria do estado econômico e financeiro, dos aspectos sociais, ambientais e tecnológicos baseiam-se na construção de soluções corporativas para a conversão simultânea e contínua do conhecimento individual em coletivo, do conhecimento estratégico em operacional e do conhecimento tácito em explícito.

\section{PROCESSOS DE GESTÃO DO CONHECIMENTO}

Nonaka e Takeuchi (1997, p. 80-81) apresentam que a interação entre o conhecimento tácito e o conhecimento explícito ocorre em quatro modos diferentes: socialização, externalização, combinação e internalização.

A socialização é a conversão do conhecimento tácito individual para o conhecimento tácito do grupo. Está associada às teorias dos processos de grupo e da cultura organizacional. Segundo Nenonen (2004, p. 235) é neste campo de interação que os indivíduos compartilhamento experiências e espaço ao mesmo tempo. Para Diakoulakis et al., (2004, p. 34), este processo inclui o compartilhando de experiências, ideias, imagens, modelos mentais e habilidades técnicas. Ocorre através de atividades em comum, observação, imitação e prática. 
A externalização é a conversão do conhecimento tácito em conhecimento explícito. É um processo de criação do conhecimento conceitual perfeito, na medida em que o conhecimento tácito se torna explícito, expresso na forma de metáforas, analogias, narrativas, conceitos, hipóteses ou modelos. O processo de criação do conceito é provocado pelo diálogo, ou pela reflexão coletiva. Diakoulakis et al., (2004, p. 34) citam o apoio das técnicas computacionais (modelo visual, mecanismos de conclusão indutiva/dedutiva, metodologias de aprendizado, estudos de caso, sistemas de apoio à decisão, etc.) capazes de auxiliar o indivíduo a descrever, expressar e explicar os seus conceitos.

A combinação é a conversão do conhecimento explícito em conhecimento explícito sistêmico. Origina-se no processamento de informações. É um processo de sistematização de conceitos em um sistema de conhecimento, combinando conjuntos diferentes de conhecimento explícito (por exemplo, documentos, reuniões, trocas de email). Diakoulakis et al., (2004, p. 34) citam que este modo é apoiado não só por tecnologias computacionais, como na externalização, mas também por meio de redes: bancos de dados, classificação, metodologias, ferramentas Web, intranets e Internet.

A internalização é a conversão do conhecimento explícito em conhecimento tácito. Relaciona- se com o aprendizado organizacional. Esta conversão é favorecida pela transferência do conhecimento explícito para demais pessoas, ajudando-as a vivenciar operacionalmente as experiências de outros. Nenonen (2004, p. 236) sugere para a internalização a utilização de vídeos institucionais e manuais, como da qualidade.

Nonaka e Takeuchi (1997, p. 79) sustentam que a criação do conhecimento organizacional é uma interação contínua e dinâmica entre o conhecimento tácito e o explícito. Essa interação é promovida continuamente na forma de uma espiral de navegação dos quatro modos apresentados. Essa espiral começa no nível individual e vai se desenvolvendo, ampliando comunidades de interação que cruzam fronteiras entre seções, departamentos, divisões e organizações.

Nonaka e Takeuchi (1997) trabalham a valorização do conhecimento tácito na fase do compartilhamento do conhecimento no modelo das cinco fases, em que ocorre 
a formação de um espaço de interação entre membros auto-organizados de vários departamentos funcionais que trabalham juntos para alcançar uma meta comum.

As condições capacitadoras, tratadas por Nonaka e Takeuchi (1997) relevantes a este trabalho são: interação, autonomia, flutuação e caos criativo, redundância e a variedade de requisitos. A intenção é a conceitualização de uma visão sobre o tipo de conhecimento que deve ser desenvolvido para a operacionalização dos planos estratégicos. Todos os agentes que interagem em uma organização devem agir de forma autônoma, conforme as deliberações da estratégia. A flutuação e o caos criativo estimulam a interação entre a organização e o ambiente externo. Na redundância, o conhecimento estratégico deve ser compartilhado por todos os agentes, independentemente de seus cargos funcionais, estimulando as práticas por meio do conhecimento tácito dos trabalhadores. Por fim, a variedade de requisitos na rede de relacionamentos deve estar orientada para reagir diante aos desafios dos elementos externos, os quais podem ameaçar a posição competitiva da empresa.

Graham e Pizzo (1998) apresentam o equilíbrio do conhecimento estratégico em dois domínios: fluído e institucional, sendo considerado como fluído o conhecimento espontâneo, criativo, dinâmico e experimental. O institucional, é o conhecimento estruturado, codificado, controlado e medido. Esse equilíbrio visa auxiliar a gerência a tornar o conhecimento uma atividade produtiva, criando um contexto social que maximiza a criatividade e a produtividade humanas.

Edvinsson e Malone (1998) trazem à luz formas de capital intelectual $(\mathrm{Cl})$ nas organizações: capital humano, capital estrutural e capital de clientes. Capital humano é toda capacidade, conhecimento, habilidade e experiência individuais dos empregados e gerentes, devendo-se incluir a criatividade e a inovação organizacional. O capital estrutural é descrito como o empowerment e a infra-estrutura que apóiam o capital humano, decomposto em três tipos: organizacional, de inovação e de processos. $O$ organizacional abrange o investimento da empresa em sistemas, instrumentos e filosofia operacional que agilizam o fluxo de conhecimento nos ambientes interno e externo à organização. $O$ de inovação refere-se à capacidade de renovação e aos resultados da inovação sob a forma de direitos comerciais amparados por lei, 
propriedade intelectual e outros ativos e talentos intangíveis utilizados para criar e colocar rapidamente no mercado novos bens e serviços. O capital do tipo de processos é constituído por aqueles processos, técnicas e programas direcionados aos empregados, para aumentar sua eficiência produtiva. Este último é o tipo de conhecimento prático empregado na criação contínua do valor. O capital de clientes ressalta 0 valor das relações mantidas com os clientes, separando-as do relacionamento empresa-empregados e parceiros estratégicos. Edvinsson e Malone (1998) estabelecem o conceito da formação de um índice único de Cl. Com o uso deste índice torna-se possível o comparativo estratégico entre diferentes tipos de empresa, favorecendo um benchmarking entre as organizações que adotam o mesmo sistema de avaliação de seus processos de Gestão do Conhecimento (GC).

Sveiby (2001) discorre sobre o conceito de transferência do conhecimento, classificando-o como ativo intangível em um grupo de três elementos: competência do funcionário, estrutura interna e estrutura externa. A competência do funcionário envolve a capacidade de tomadas de ações em diversas situações para criar ativos tangíveis e intangíveis para a organização. A estrutura interna inclui patentes, conceitos, modelos, cultura organizacional e sistemas administrativos e computacionais. A estrutura externa inclui as relações com clientes e fornecedores, marcas registradas e a imagem da organização.

Sveiby (2001) e Arora (2000) consideram a importância da análise de competências para a execução de atividades que requerem conhecimentos específicos. As informações dessa análise podem ser cruzadas com os objetivos estratégicos em busca do alcance das metas de cada colaborador. Deste modo, pode-se identificar as lacunas de competências que devem ser preenchidas para que essas metas sejam operacionalizadas. De acordo com esses autores, essas lacunas podem ser preenchidas pela aquisição de conhecimento externo (por exemplo: especialistas, parceiros, licença para uso de patentes) ou ainda, pelo desenvolvimento das competências estratégicas internas (por meio de seus próprios colaboradores).

Probst, Raub e Romhardt (2002) descrevem os processos de GC em elementos construtivos do conhecimento: objetivo, identificação, aquisição, desenvolvimento, 
disseminação, utilização retenção e avaliação. De acordo com os mesmos, o objetivo deve representar a transformação dos objetivos estratégicos em termos de conhecimento. A identificação é a visibilidade das lacunas do conhecimento para ajudar as organizações a optarem entre adquirir ou desenvolvê-lo internamente. A aquisição distingue entre a aquisição de conhecimento diretamente utilizável daquele potencial de conhecimento. $O$ desenvolvimento é a vinculação dos objetivos de conhecimento ao seu processo de criação. A disseminação é o entrelaçamento da empresa com seu ambiente, minimizando as formas de trabalho individual e valorizando as formas coletivas. A utilização é a fase de implementação do processo de GC. A retenção divide-se em três fases: seleção; armazenagem e atualização do conhecimento. A avaliação significa decidir se os objetivos de conhecimento foram ou não atingidos.

Davenport e Prusak (1998) afirmam que os espaços de transferência do conhecimento são outro processo de interesse para a aplicação de um sistema de GC. As conversas que ocorrem entre colaboradores nos bebedouros ou restaurantes da empresa não são consideradas como perda de tempo, pois enriquecem a troca de experiências no trabalho. Para favorecer o compartilhamento do conhecimento tácito dos trabalhadores, os autores apresentam um exemplo de empresas japonesas que utilizam o sistema de rodízio de executivos para a produção e vice-versa, de forma a promover que os gerentes compreendam todo o processo de desenvolvimento e de fabricação dos produtos.

Grotto (2002, p. 113) e Pereira (2002) ressaltam a importância da explicitação das melhores práticas para o desenvolvimento de planos de ações que visem o alcance dos objetivos da organização. A descrição das melhores práticas deve estar facilmente disponível aos trabalhadores, sendo armazenados eletronicamente para consultas posteriores. Os autores referem-se a este armazenamento como "páginas amarelas" ou "mapas do conhecimento".

Arora (2002) cita que a priorização dos objetivos da GC pode ser feita atribuindose pesos para eles, de acordo com o grau de importância e relevância. O autor sintetiza esta atividade em quatro passos: (a) dar peso de importância para cada objetivo estratégico da GC; (b) correlacionar cada objetivo da GC aos objetivos estratégicos; (c) 
selecionar os parâmetros com máximo peso global; e (d) desenvolver um índice de GC global.

Diakoulakis et al., (2004) identificam relações de causa e efeito entre os seguintes processos de GC: retenção/sistematização do conhecimento, acesso compartilhado ao conhecimento, combinação/criação do conhecimento, exploração do contexto externo, decomposição do contexto interno e uso do conhecimento. Essas relações causais são consideradas como forças diferentes que iniciam nos indicadores gerenciais e terminam nos processos de GC. Acrescentam, ainda que os objetivos estabelecidos pelas organizações para satisfazer a GC são multidimensionais.

Powell e Swart (2005) apresentam uma técnica que captura as qualidades dinâmicas e sistêmicas de conhecimento sob quatro formas de saber: o que saber, como saber, por que saber e quem sabe; examinando o contexto de sistema no qual o conhecimento é usado e os papéis de seus usuários.

Schreiber (2004) explica que o modelo CommonKADS é uma metodologia estruturada para apoiar a engenharia do conhecimento. O CommonKADS permite identificar as oportunidades e problemas dentro das organizações, trazendo informações de como as empresas desenvolvem, distribuem e aplicam os recursos do conhecimento. O CommonKADS também provê os métodos para executar uma análise detalhada de processos e tarefas relacionados ao conhecimento. Por fim, apóia o desenvolvimento de sistemas de conhecimento orientados para o planejamento das necessidades de mudanças organizacionais.

Santiago Jr. (2004) pauta em sua metodologia a necessidade da localização do conhecimento na empresa para tornar possível a sua utilização. Segundo esse autor, o mapa do conhecimento "revela pontos fortes a serem explorados e as lacunas a serem preenchidas", indicando para cada colaborador suas habilidades e competências, "conforme o grau de conhecimento sobre cada uma das áreas de atuação da empresa". Melo e Sattamini (2005) e ABIPTI / FINEP - FNDCT / CT (2005) citam que as comunidades de especialistas têm como objetivo desenvolver facilidades de comunicação entre os agentes que compartilham as mesmas práticas, interesses ou objetivos de trabalho. Este intercâmbio pode se dar por meio de correio eletrônico, informativos, reuniões, grupos de discussão, ou 
acesso a documentos e banco de dados. Podem ser incluídas como fonte de informações: relatórios gerenciais, relatórios de projetos, atas de reuniões, apresentações, legislação, normas e regulamentos técnicos, artigos de periódicos, artigos em anais de congressos, teses e estudos de caso.

Terra (2005) aborda as políticas de recursos humanos, afirmando que as mesmas devem estar associadas à aquisição de conhecimentos externos e internos, à geração, difusão e armazenamento de conhecimentos. Esse autor destaca as seguintes iniciativas: processos seletivos de contratação rigorosos; planos de carreira e capacitação que ampliam as experiências individuais e coletivas; e esquemas de remuneração associados à aquisição de competências individuais, ao desempenho da equipe e da empresa no curto e longo prazo.

Outro processo de GC enfatizado por Terra (2005) é a relevância da cultura organizacional, pois é o que torna o aprendizado, a colaboração e o compartilhamento de conhecimentos (tácitos ou explícitos) parte da rotina dos colaboradores, das estratégias e dos investimentos em infra-estrutura. Para esse autor, na GC o desenvolvimento de uma cultura organizacional voltada à inovação, experimentação, aprendizado contínuo, comprometimento com os resultados de longo prazo e com a otimização dos processos é fundamental para a alta administração.

Em busca da vinculação destes processos de GC selecionados, se faz necessário o desenvolvimento de um modelo gerencial dinâmico que atenda a estas necessidades, sob uma abordagem dialética, conforme proposto neste artigo. De acordo com Gil (1999, p. 26), o método dialético fundamenta-se na proposta de Hegel, na qual as contradições se transcendem dando origem a novas contradições que passam a requerer solução. É um método de interpretação dinâmica e totalizante da realidade. Considera que os fatos não podem ser analisados fora de um contexto social, político e econômico.

Segundo Dagnino (2002, p. 96), a construção de um modelo é essencial para se compreender o funcionamento do sistema. Desta forma, é possível atuar sobre cada uma de suas características. Assim, a próxima seção deste artigo apresenta um modelo para o alinhamento da GC ao BSC. O mesmo foi elaborado considerando a modelagem 
sistêmica e buscando contribuir na construção de soluções corporativas para a conversão simultânea e contínua do conhecimento individual em coletivo, do conhecimento estratégico em operacional e do conhecimento tácito em explícito.

\section{MODELO DE ALINHAMENTO DA GC AO BSC}

A estrutura do modelo proposto para o alinhamento da GC ao BSC norteia-se em um movimento contínuo das dimensões da conversão do conhecimento. A sua força dinâmica é produzida pela progressão e retroalimentação de cinco fases que combinam os processos de GC aos propósitos do BSC.

O modelo contempla a rede de relacionamento entre os agentes estratégicos do conhecimento organizacional inseridos nos ambientes interno (funcionários; clientes, fornecedores, distribuidores, bancos e órgão fomentador etc.) e globalizado (governo, sociedade, tecnologia, consultores, sindicatos etc.) do conhecimento cognitivo (combinação entre o público e o privado, sendo transferido e compartilhado com o mercado) e do conhecimento conectivo (inserido nos sistemas organizacionais, criado por indivíduos separadamente).

A Figura 1 apresenta a dinâmica das cinco fases que compõem o modelo proposto. O movimento entre estas fases ocorre por um efeito de respostas à ação do próprio sistema. 


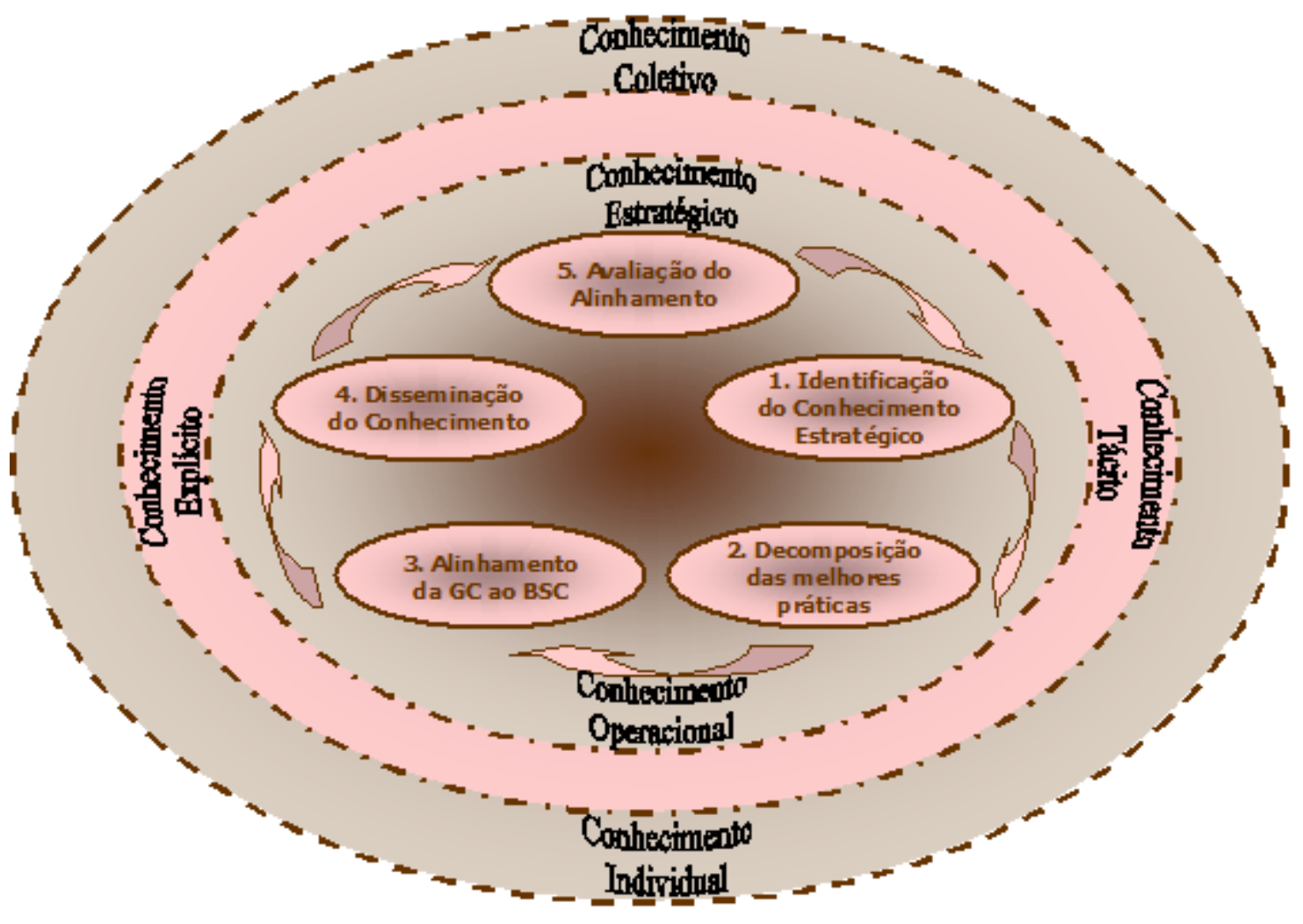

Figura 1 - Dinâmica das cinco fases do modelo de alinhamento da GC ao BSC

As fases do modelo são constituídas dos seguintes elementos:

- Fase 1 - Identificação do conhecimento estratégico: identifica os parâmetros do conhecimento requeridos pelos propósitos do BSC;

- Fase 2 - Decomposição das melhores práticas: cria uma cadeia de causalidade entre as variáveis da GC e os objetivos do BSC;

- Fase 3 - Alinhamento da GC ao BSC: estrutura o sistema de informação para apoiar o alinhamento da GC ao BSC;

- Fase 4 - Disseminação do conhecimento: constrói/atualiza a rede de interação para promover a partilha das melhores práticas requeridas para o alcance dos resultados estratégicos; $\mathrm{e}$

- Fase 5 - Avaliação do sistema de alinhamento da GC ao BSC: analisa o uso do conhecimento estratégico e promove a avaliação da conversão do conhecimento sobre os elementos do conhecimento. 
Uma proposta para integrar a gestão do conhecimento ao Balanced Scorecard

Simone de Cássia Silva, Paulo Mauricio Selig, José Luís Duarte Ribeiro

As cinco fases são desdobradas em dez etapas que visam promover a atualização permanente da conversão do conhecimento requerida para que os agentes executem com sucesso os objetivos estratégicos da organização. O Quadro 1 visa ordenar a sua estruturação, apresentando para cada uma das fases as suas etapas e seus respectivos objetivos.

\begin{tabular}{|c|c|c|}
\hline Fase & Etapa & Objetivo \\
\hline \multirow[b]{2}{*}{$\begin{array}{l}1 \text { - Identificação do } \\
\text { conhecimento estratégico }\end{array}$} & $\begin{array}{l}1.1 \text { - Analisar os objetivos } \\
\text { estratégicos do BSC }\end{array}$ & $\begin{array}{l}\text { Compreender os elementos das diretrizes } \\
\text { estratégicas da empresa }\end{array}$ \\
\hline & $\begin{array}{l}1.2 \text { - Identificar problemas e } \\
\text { oportunidades }\end{array}$ & $\begin{array}{l}\text { Propor soluções aos problemas e } \\
\text { oportunidades identificados nas lacunas do } \\
\text { conhecimento estratégico }\end{array}$ \\
\hline \multirow{2}{*}{$\begin{array}{l}2 \text { - Decomposição das } \\
\text { melhores práticas }\end{array}$} & \begin{tabular}{|l|}
2.1 - Identificar os membros \\
da rede de relacionamento do \\
processo
\end{tabular} & $\begin{array}{l}\text { Verificar o grau de cooperação entre as } \\
\text { áreas de foco do conhecimento e os } \\
\text { membros da rede de relacionamento }\end{array}$ \\
\hline & $\begin{array}{l}2.2 \text { - Decompor as atribuições } \\
\text { dos agentes do conhecimento }\end{array}$ & $\begin{array}{l}\text { Explicitar as faculdades de cada agente do } \\
\text { conhecimento no sistema }\end{array}$ \\
\hline \multirow{2}{*}{$\begin{array}{l}\text { 3-Alinhamento da GC ao } \\
\text { BSC }\end{array}$} & $\begin{array}{l}3.1 \text { - Estruturar a cadeia de } \\
\text { causalidade das variáveis da } \\
\text { GC }\end{array}$ & $\begin{array}{l}\text { Identificar as variáveis endógenas e } \\
\text { exógenas da GC e estruturar a cadeia de } \\
\text { causalidade entre as variáveis do sistema }\end{array}$ \\
\hline & $\begin{array}{l}3.2 \text { - Integrar a visão gerencial } \\
\text { ao sistema }\end{array}$ & $\begin{array}{l}\text { Organizar as informações do alinhamento } \\
\text { global para tomadas de decisão }\end{array}$ \\
\hline \multirow{2}{*}{$\begin{array}{l}4 \text { - Disseminação do } \\
\text { conhecimento }\end{array}$} & $\begin{array}{l}4.1 \text { - Identificar a viabilidade } \\
\text { do projeto de alinhamento }\end{array}$ & $\begin{array}{l}\text { Analisar a viabilidade do negócio, a } \\
\text { viabilidade técnica do projeto de melhoria do } \\
\text { sistema e as ações propostas }\end{array}$ \\
\hline & $\begin{array}{l}\text { 4.2 - Compartilhar o sistema } \\
\text { de alinhamento da GC ao } \\
\text { BSC }\end{array}$ & $\begin{array}{l}\text { Desenvolver facilidades de comunicação e } \\
\text { uso do conhecimento }\end{array}$ \\
\hline \multirow{2}{*}{$\begin{array}{l}5 \text { - Avaliação do sistema } \\
\text { de alinhamento da GC ao } \\
\text { BSC }\end{array}$} & $\begin{array}{l}5.1 \text { - Promover o feedback do } \\
\text { conhecimento requerido pelo } \\
\text { BSC }\end{array}$ & $\begin{array}{l}\text { Avaliar os resultados do sistema e os efeitos } \\
\text { da conversão do conhecimento }\end{array}$ \\
\hline & $\begin{array}{l}5.2 \text { - Avaliar os impactos e } \\
\text { melhorias }\end{array}$ & $\begin{array}{l}\text { Avaliar os impactos e melhorias na } \\
\text { organização com o uso do sistema de } \\
\text { alinhamento }\end{array}$ \\
\hline
\end{tabular}

Quadro 1 - Descrição das etapas do modelo de alinhamento da GC ao BSC

As dez etapas são explicitadas pelo uso do modelo CommonKADS. Schreiber (2004) explica que esse modelo foi estruturado para apoiar a engenharia do conhecimento. Pacheco (2005) afirma que a Engenharia do Conhecimento (EC) "é uma atividade construtiva e colaborativa em que a modelagem é o aspecto central", e que a "EC é uma metodologia que tem como produto principal sistemas de conhecimento". 
Esse autor cita como benefícios da EC a identificação das áreas de possível aplicação para a GC e o destaque das áreas que constituem gargalos de conhecimento.

\subsection{Etapa 1.1 - Analisar os objetivos estratégicos do BSC}

A primeira etapa do modelo proposto tem como objetivo compreender os elementos das diretrizes estratégicas da empresa.

Para o alcance do propósito desta etapa, é necessário contextualizar o cenário estratégico em que o BSC está inserido. Analisam-se os processos históricos numa visão holística da base de conhecimento implícita nos elementos da cultura organizacional: a missão, valores, visão e estratégia da organização. A seguir, busca-se compreender as relações de causa e efeito entre os objetivos espelhados no mapa estratégico do BSC. O resultado deverá clarificar quais são os conhecimentos requeridos aos agentes para a execução de suas atividades, conforme as demandas estratégicas da organização. Esta atividade inicia a modelagem do sistema de engenharia do conhecimento do modelo de gestão para o alinhamento da GC ao BSC.

\subsection{Etapa 1.2 - Identificar problemas e oportunidades}

Esta etapa tem como objetivo propor soluções aos problemas e oportunidades identificados nas lacunas do conhecimento estratégico.

A partir desta etapa utiliza-se a técnica de construção de cenários para a dinamização e encadeamento entre os níveis macro, meso e micro, onde a gestão estratégica irá atuar. Mediante a construção de hipóteses plausíveis acerca do futuro, pode-se identificar as implicações na base de conhecimento dos trabalhadores sobre 0 nível das informações estratégicas. Para esta atividade, identificam-se as melhores práticas requeridas para o alcance da operacionalização dos objetivos de cada perspectiva do BSC. Observa-se, ao fim desta etapa, que o modelo proposto evidencia a forma e o método analítico do conhecimento, promovendo a interatividade entre o conhecimento estratégico e o operacional (chamado "melhores práticas"). Inicia-se, na sequência, o dinamismo da conversão do conhecimento tácito em conhecimento explícito. 


\subsection{Etapa 2.1 - Identificar os Membros da Rede de Relacionamento do Processo}

Esta etapa tem como objetivo verificar o grau de cooperação entre as áreas de foco do conhecimento e os membros da rede de relacionamento.

A partir da lista das melhores práticas requeridas pelo BSC, é possível identificar os membros da rede de relacionamento que irão interagir diretamente com a aquisição e disseminação do conhecimento da empresa, aqueles co-responsáveis pelo compartilhamento do conhecimento estratégico. Com base nestas informações, utilizase a técnica de diagnóstico de situações. A análise permite identificar os graus de cooperação entre as áreas de foco das melhores práticas requeridas pelo BSC e os potenciais agentes do conhecimento, adotando-se como referencial os seguintes elementos: tempo (atuais ou potenciais); governabilidade (controle total, baixo controle e fora de controle); abrangência (nacionais, locais, específicos, estaduais e municipais); e estruturação (estruturados ou quase-estruturados), baseado em Dagnino (2002, p. 85).

\subsection{Etapa 2.2 - Organizar as Atribuições dos Agentes do Conhecimento}

O objetivo desta etapa é explicitar as faculdades de cada agente do conhecimento no sistema, utilizando como instrumento a Planilha de Descrição dos Agentes (AM-1) do CommonKADS. Desenvolve-se uma ferramenta para cada agente inserido no sistema de alinhamento da GC ao BSC, identificando seu nome, função na rede de relacionamento, lista dos objetivos estratégicos em que está envolvido, comunicação com demais integrantes da rede, conhecimento e outras competências, ou seja, as melhores práticas que requerem a participação do agente dentro do sistema e, por fim, responsabilidades e restrições, descrevendo quais são seus indicadores de desempenho para o monitoramento da consecução dos objetivos estratégicos, bem como suas limitações na autoridade, normas legal-profissionais ou regras afins. No desenvolvimento desta fase, já se observa que as ferramentas, além de serem utilizadas como instrumento de apoio para a engenharia do conhecimento, também dinamizam os vetores da conversão do conhecimento individual em coletivo e do conhecimento estratégico em operacional. 


\subsection{Etapa 3.1 - Estruturar a Cadeia de Causalidade das Variáveis da GC}

Os objetivos desta etapa são identificar as variáveis endógenas e exógenas da GC e estruturar a cadeia de causalidade entre as variáveis do sistema.

Para atender este propósito retorna-se ao uso da técnica de análise estrutural. Nesta técnica consideram-se as melhores práticas como variáveis endógenas do sistema de alinhamento da GC ao BSC, enquanto as variáveis de cooperação (tempo, governabilidade, abrangência e estruturação) são tratadas como variáveis exógenas. $\mathrm{O}$ produto da análise de motricidade- dependência é a estruturação primária da cadeia de causalidade das variáveis que irão compor o sistema de alinhamento, chamada neste modelo de mapa do conhecimento. De acordo com a técnica da análise estrutural, o objetivo da avaliação do mapa do conhecimento tem em vista não apenas descrever e explicar o alinhamento da GC ao BSC (modelagem), mas identificar seu potencial impacto sobre a trajetória futura do alinhamento deste sistema.

\subsection{Etapa 3.2 - Integrar a Visão Gerencial ao Sistema}

O objetivo desta etapa é organizar as informações do alinhamento global para subsidiar o processo de tomada de decisão.

Esta etapa implementa um plano de ação para o alinhamento da GC ao BSC por meio de um controle gerencial da melhor prática, que deve reunir as seguintes informações: responsável, documentos relacionados, indicadores, metas e custos de implementação.

O ordenamento desta fase evidencia a modelagem para a "justificação das cognições". Verifica-se, ainda, conforme a teoria de Polanyi, o lado subjetivo à ciência, o conhecimento tácito, "o elemento intuitivo do saber" - fator observado no processo de filtragem da técnica de análise estrutural ao se desenvolver o mapa do conhecimento da organização.

\subsection{Etapa 4.1 - Identificar a Viabilidade do Projeto de Alinhamento}

O objetivo desta etapa é analisar a viabilidade do negócio, a viabilidade técnica do projeto de melhoria do sistema e as ações propostas. 
Esta etapa utiliza como ferramenta a Planilha de Análise de Viabilidade de Solução (OM-5) do CommonKADS. Este instrumento consiste em sintetizar todos os elementos-chave do modelo em um único documento, partindo da seguinte questão: Qual é a área de oportunidades mais promissora para aplicações, e qual é a melhor direção de solução? A Planilha OM-5 organiza os seguintes elementos de resposta à referida questão: viabilidade do negócio, viabilidade técnica, viabilidade do projeto e ações propostas.

\subsection{Etapa 4.2 - Compartilhar o Sistema de Alinhamento da GC ao BSC}

Esta etapa conclui a Fase 4 do modelo, tendo como objetivo desenvolver facilidades de comunicação e uso do conhecimento.

A utilização da base de conhecimento sistêmico deve tornar o compartilhamento e a distribuição de conhecimento uma pré-condição para uma gestão coletiva. $\mathrm{O}$ compartilhamento do conhecimento sistêmico pode ser facilitado por meio da "Comunidade Virtual". A sinergia entre os eixos filosóficos deve manter o dinamismo do BSC para agilizar a disseminação do conhecimento. Para o alcance deste objetivo, é necessário compartilhar as informações contidas no sistema e, sobretudo, estimular os agentes na sua utilização e atualizações, para que os resultados desejados possam ser harmonicamente alcançados. Ambilateralmente, o conhecimento flui entre os níveis individual e coletivo, entre as diretrizes estratégica e operacional e entre os campos tácito e explícito. Tendo-se delineado os procedimentos operacionais para a utilização do conhecimento estratégico, a fase apresentada a seguir descreve a forma de avaliação da efetividade deste sistema.

\subsection{Etapa 5.1 - Promover o Feedback do Conhecimento Requerido pelo BSC}

Esta etapa do modelo tem como objetivo avaliar os resultados do sistema e os efeitos da conversão do conhecimento.

A Figura 2 representa a estrutura do sistema de avaliação do alinhamento da GC ao BSC, baseada na adaptação do elemento construtivo de Probst, Raub e Romhardt (2002). Os indicadores de desempenho do sistema de alinhamento da GC ao BSC são 
organizados em três classes distintas: classe I (medem os resultados do BSC); classe II (medem os graus de cooperação entre os membros da rede de relacionamento da empresa e as áreas de foco do conhecimento estratégico); e classe III (medem o desempenho das melhores práticas).

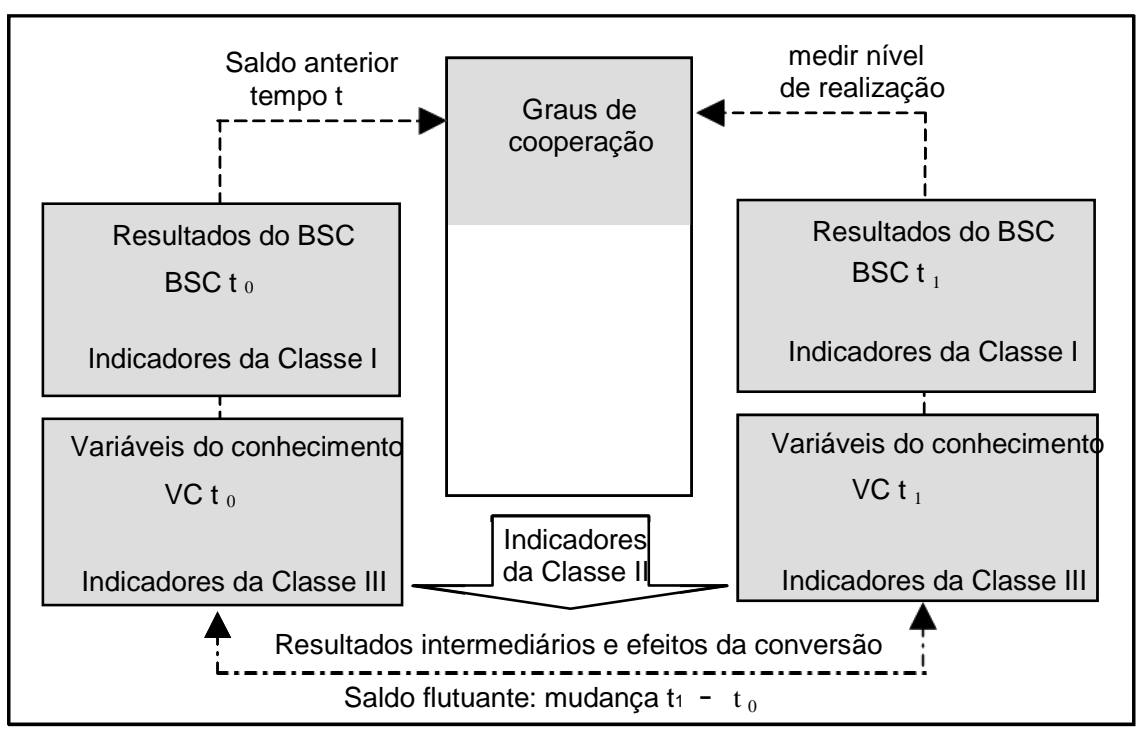

Figura 2 - Estrutura do sistema de avaliação do alinhamento da GC ao BSC.

Fonte: baseado em Probst, Raub e Romhardt (2002, p. 205)

Os indicadores das Classes I, II e III devem estar organizados em ferramentas que incluem o monitoramento das causas dos desvios entre as metas planejadas e as metas realizadas.

Baseando-se no processo de "loop duplo", mencionado por Kaplan e Norton (2000, p. 290), esta etapa integra a GC e das operações com a gestão estratégica, sistematizando o dinamismo do feedback de conhecimento requerido pelo BSC. Considera-se que as empresas que utilizam o BSC estejam integrando a gestão orçamentária à gestão estratégica, de acordo com a metodologia do próprio sistema estratégico.

Adaptando os conceitos de Kaplan e Norton (2000, p. 289), os resultados dos indicadores de classe I e III devem permitir o monitoramento do progresso em relação às metas estratégicas, originárias do orçamento. Eles também permitem a 
adoção das ações corretivas necessárias à implementação das melhores práticas. Então, o loop dos resultados estratégicos ocorre pela atualização da estratégia em razão das mudanças nos ambientes, bem como da análise da viabilidade do projeto. O loop dos eixos filosóficos é o resultado do sistema de avaliação do alinhamento da GC ao BSC e a necessidade dos ajustes deste alinhamento.

\subsection{Etapa 5.2 - Avaliar os Impactos e Melhorias}

Esta etapa tem como objetivo avaliar os impactos e melhorias na organização com o uso do sistema de alinhamento.

Para a sua execução, esta etapa utiliza como ferramenta a Planilha com Checklist de Impactos e Melhorias (OTA-1) do CommonKADS, que reúne as informações necessárias para tomadas de decisão sobre as mudanças e melhorias trazidas pelo uso do sistema de alinhamento. Por isto, esta ferramenta deve ser utilizada após o feedback dos primeiros resultados trazidos pelo sistema, incorporando os seguintes elementos: impactos e mudanças na organização, impactos e mudanças específicos a tarefas/agentes, atitudes e compromissos e ações propostas. Uma vez sistematizado o alinhamento, a próxima atividade é compartilhá-lo por toda organização, atentando-se à necessidade de atualizações. Desta forma, a Etapa 5.2 encerra o descritivo da implementação do modelo e renova um o ciclo do alinhamento da GC ao BSC, por meio da análise dos resultados do sistema de avaliação.

\section{CONSIDERAÇÕES FINAIS}

Este artigo considerou a identificação, seleção, disseminação e avaliação central de informação como atividade principal para o desenvolvimento do conhecimento estratégico, coletivo e explícito. Neste contexto, as organizações devem a ser vistas como grupos de comunicação de redes interligadas, concluindo-se que o conceito conectivo direciona o conhecimento às conexões da rede de relacionamento estratégico e, consequentemente, focaliza-se no fluxo de informação organizado coletivamente. Assim, deve-se entender que o conhecimento nas organizações não pode ser moldado 
em parâmetros predeterminados considerando-se os entes fixos, pois o processamento das informações varia de acordo com os interesses de cada stakeholder, e, ainda, com cada cultura organizacional.

Foi apresentado um modelo para o alinhamento da gestão do conhecimento ao Balanced Scorecard. O modelo proposto está organizado em cinco fases, que contemplam: (i) identificação do conhecimento estratégico, (ii) decomposição das melhores práticas, (iii) alinhamento da GC ao BSC, (iv) disseminação do conhecimento e (v) avaliação do sistema de alinhamento da GC ao BSC. As cinco fases são desdobradas em dez etapas que visam promover a atualização permanente da conversão do conhecimento requerida para que os agentes executem com sucesso os objetivos estratégicos da organização.

\section{REFERÊNCIAS}

ABIPTI/FINEP - FNDCT/CT - Verde Amarelo. Comunidades de prática/portais: projeto rede de centros especializados em gestão tecnológica. Disponível em: http://www.abipti.org.br, acesso em 13/02/2007.

ARORA, R. 2002. Implementing KM - a balanced score card approach. Journal of Knowledge Management. 6 (3): 240-249.

DAGNINO, R. 2002. Gestão estratégica da inovação: metodologias para análise e implementação. Taubaté, Cabral Editora e Livraria Universitária, p. 85-96.

DAVENPORT, T. e PRUSAK, L. 1998. Conhecimento empresarial: como as organizações gerenciam o seu capital intelectual. Rio de Janeiro, Campus, 256 p.

DIAKOULAKIS, I. E.; GEORGOPOULOS, N. B.; KOULOURIOTIS, D. E. e EMIRIS, D. M. 2004. Towards a holistic knowledge management model. Journal of Knowledge Management. 8(1):32-46.

EDVINSSON, Leif e MALONE, Michael S. 1998. Capital intelectual: descobrindo o valor real de sua empresa pela identificação de seus valores internos. São Paulo, Makron Books, $214 \mathrm{p}$.

GIL, A. C. 1999. Métodos e técnicas de pesquisa social. São Paulo, Atlas, 208 p.

GRAHAM, A. B. e PIZZO, V. G. 1998. Uma questão de equilíbrio: estudos de casos na gestão estratégica do conhecimento. In: KLEIN, D. A. A estratégia do capital intelectual: recursos para a economia baseada em conhecimento. Rio de Janeiro: Qualimark, p. 16- 
39.

GROTTO, Daniela. 2002. O compartilhamento do conhecimento nas organizações. In: ANGELONI, Maria Terezinha (coord.). Organizações do conhecimento: infra-estrutura, pessoas e tecnologias. São Paulo, Saraiva, p. 113.

KAPLAN, R. S. e NORTON, D. P. 2000. Organização orientada para a estratégia: como as empresas que adotam o balanced scorecard prosperam no novo ambiente de negócios. Rio de Janeiro, Campus, 416 p.

MELO, Marcelo P. e SATTAMINI, Suzana R. A. Criação de comunidades virtuais na Petrobrás. Disponível em: http://www.abed.org.br/antiga/htdocs/paper_visem/marcelo_pereira_melo, acesso em: 02/03/2007.

NENONEN, S. 2004. Analysing the intangible benefits of work space. Facilities. 22(9/10):233-239.

NONAKA, I. e TAKEUCHI, H. 1997. Criação de conhecimento na empresa. Rio de Janeiro, Campus, 1997. 380 p.

PACHECO, R. C. S. 2005. Engenharia do conhecimento. Notas de aulas do Programa de Pós-Graduação em Engenharia e Gestão do Conhecimento da Universidade Federal de Santa Catarina.

PEREIRA, R.C.F. 2002. As redes como tecnologias de apoio à gestão do conhecimento. In ANGELONI, Maria Terezinha. (coord.). Organizações do conhecimento: infra-estrutura, pessoas e tecnologias. São Paulo, Saraiva, p. 62-78.

POWELL, J.H. e SWART, J. 2005. This is what the fuss is about: a systemic modelling for organisational knowing. Journal of Knowledge Management, 9(2):45-58.

PROBST, G.; RAUB, S. e ROMHARDT, K. 2002. Gestão do conhecimento: os elementos construtivos do sucesso. Porto Alegre, Bookman, $286 \mathrm{p}$.

SANTIAGO JR., J. R. S. 2004. Gestão do conhecimento: a chave para o sucesso empresarial. São Paulo, Novatec, 208 p.

Data de Submissão: 14/12/2007

Data de Aceite: 15/12/2007 